\title{
Studies of Styrene and 2-Vinylpyridine Block Copolymers; Preparation and Characterization
}

\author{
Yushu Matsushita, Yasushi NaKaO, Ryuichi SAGUCHI, \\ Haruhisa CHOSHI, and Mitsuru NAGASAWA \\ Department of Synthetic Chemistry, Nagoya University, \\ Furo-cho, Chikusa-ku, Nagoya 464, Japan
}

(Received December 16, 1985)

\begin{abstract}
Preparation of di- and triblock copolymers of styrene- $h_{8}$, styrene- $d_{8}$, and 2vinylpyridine by anionic polymerization was carried out for the purpose of obtaining block copolymer samples containing a poly(styrene- $d_{8}$ ) part and having well defined molecular characteristics. The block copolymerization proceeded successfully when the polymerization was carried out in the order of styrene- $h_{8}$, styrene- $d_{8}$, and 2-vinylpyridine or of styrene- $d_{8}$, styrene- $h_{8}$, and 2vinylpyridine, whereas it was not successful when the polymerization was started from 2vinylpyridine. All block copolymer samples polymerized in the order of styrene and 2-vinylpyridine were found to have narrow molecular weight distributions with respect to each component.

KEY WORDS Block Copolymer / Anionic Polymerization / Narrow

Molecular Weight Distribution / Deuterated Polystyrene / Styrene-2-

Vinylpyridine Block Copolymer /
\end{abstract}

Triblock copolymers containing a deuterated polystyrene part as a component may be highly useful since the small angle neutron scattering from the deuterated part can give unique information concerning conformation of polymer chains. Because of difficulties in preparation, however, works with such triblock copolymers are scarce. Matsushita et al. ${ }^{1}$ reported the preparation of triblock copolymers of $h_{8}-d_{8}-h_{8}$ polystyrene, which were used to study the radius of gyration of a part of a homopolymer chain in dilute solution. ${ }^{2}$ In this paper, we report the preparation and characterization of di- and triblock copolymers of styrene- $h_{8}$, styrene- $d_{8}$, and 2-vinylpyridine for studying the conformation of a part of a chain locating at an interface between two different polymer domains in bulk. We chose 2-vinylpyridine as a component since the coherent scattering amplitude per unit volume of 2 vinylpyridine lies between those of styrene- $h_{8}$ and styrene- $d_{8}$.
Block copolymers of styrene and 2-vinylpyridine may have additional interest from the fact that one component is a highly polar monomer. Although quite a few studies of block copolymers have been published, studies on block copolymers consisting of nonpolar and polar monomers are not so many ${ }^{3-5}$ and their properties have not yet been fully clarified, because of difficulties in preparing the samples having well defined structures. Undesirable side reactions often occur in the anionic polymerization of polar monomers, such as methyl methacrylate ${ }^{6}$ and 4-vinylpyridine. ${ }^{7.8}$ On the other hand, it was previously confirmed that the anionic polymerization of 2-vinylpyridine proceeds in a living manner and can give samples with narrow molecular weight distributions over a wide range of molecular weight. ${ }^{9}$ 


\section{EXPERIMENTAL}

\section{Polymerization}

The block copolymer samples were prepared in vacuo at $-78^{\circ} \mathrm{C}$ by a sequential monomer addition technique in anionic polymerization. Styrene and 2-vinylpyridine were purified using triphenylmethyllithium and the dipotassium salt of $\alpha$-methylstyrene tetramer, respectively, by the same methods as reported in previous papers. ${ }^{4.9}$ Deuterated styrene of Aldrich Chemical Co. was also purified by the same method as styrene- $h_{8}$. It was pointed out in a previous paper ${ }^{1}$ that commercial styrene$d_{8}$ may contain impurities different from those in commercial styrene- $h_{8}$ and removal of the undesired impurities from styrene- $d_{8}$ is most important for carrying out its anionic living polymerization successfully. The use of triphenylmethyllithium for purification of styrene- $d_{8}$ was found to be effective, ${ }^{10}$ though a fairly large amount of the monomer is wasted.

The initiators used were cumylpotassium, cumylcesium and $n$-butyllithium, which were prepared by the same methods as previously. ${ }^{9}$ The solvent, THF, was also purified as reported previously. ${ }^{11}$ The block copolymerization was carried out in the order of styrene and 2vinylpyridine in most cases, but a copolymerization was carried out in the order of 2vinylpyridine and styrene for comparison. Before adding the second or third monomer, a portion of the polymerization mixture was sealed off to determine the molecular weights and molecular weight distributions of precursors. The active chain ends were terminated with methanol. The block copolymers thus prepared were precipitated from THF solutions with $n$-hexane. No further purification was given to the samples.

\section{Characterization}

The number-averaged molecular weights of block copolymers prepared and their precursors were determined in THF and toluene, respectively, at $25^{\circ} \mathrm{C}$, using a Hewlett Packard
Type 502 High Speed Membrane Osmometer. The apparent weight-averaged molecular weights of the samples with high molecular weights were estimated by light scattering in a single solvent (benzene) using a Fica-50 Automatic Light Scattering Photometer. A uniform refractive index increment value for polystyrene $\left(\partial_{n} / \partial_{c}=0.111\right)^{12}$ was assumed for all block copolymers. Since the difference between $\partial_{n} / \partial_{c}$ of polystyrene and that of poly(2-vinylpyridine) in benzene is small ${ }^{12.13}$ and also the molecular weight distributions of each component in the block copolymers are narrow, the apparent weight-averaged molecular weight determined in benzene alone may be close to the true weight-averaged molecular weight.

GPC chromatograms of samples were determined in THF using a Toyo-Soda HLC$802 \mathrm{~A}$ with high resolution columns (GMHXL $\times 2)$. The exclusion limit of the columns is sufficiently high $\left(4 \times 10^{8}\right.$ according to the manufacturer). $N, N, N^{\prime}, N^{\prime}$-Tetramethylethylenediamine (TMEDA) was added to the eluent, giving a $5 \%$ solution, to prevent adsorption of poly(2-vinylpyridine) on polystyrene gels. ${ }^{9}$ The GPC apparatus was calibrated with a series of Pressure Chemical Standard Polystyrenes in the same solvent. The polydispersity index $M_{w} / M_{n}$ was calculated from GPC chromatograms assuming that the calibration curve is applicable to the present block copolymers. $M_{w} / M_{n}$ thus determined are believed to be close to true ones since THF is a common good solvent for both polystyrene and poly (2vinylpyridine). Chemical compositions of the block copolymers were determined by elemental analysis, and deuterated polystyrene content of the triblock copolymers was determined from pyrolysis-gas chromatograms of polystyrene precursors. ${ }^{14}$

\section{Morphology}

The morphologies of the sample films were examined by a conventional method using a Hitachi type HU-12 transmission electron mi- 
croscope. That is, the samples were cast from $4-8 \mathrm{wt} \%$ THF (a common good solvent) solutions into thin films of about $0.15 \mathrm{~mm}$ thickness on mercury at room temperature. The films obtained were completely dried under a reduced pressure of $10^{-3} \mathrm{mmHg}$ at room tem- perature for 4 days and were stained with osmium tetroxide. The stained films were embedded in an epoxy resin, cut into ultra thin sections by an ultramicrotome and examined by the electron microscope.

Table I. Polymerization conditions

\begin{tabular}{|c|c|c|c|c|c|c|c|}
\hline \multicolumn{2}{|c|}{$\begin{array}{c}\text { Sample } \\
\text { code }^{\mathrm{a}}\end{array}$} & \multirow[t]{2}{*}{ Initiator } & \multirow{2}{*}{$\frac{\begin{array}{c}\text { Polym. } \\
\text { time }\end{array}}{h}$} & \multirow{2}{*}{$\frac{\begin{array}{c}\text { Monomer } \\
\text { concn }\end{array}}{\text { mol l-1 }^{-1}}$} & \multirow{2}{*}{$\frac{\begin{array}{c}\text { Initiator } \\
\text { concn }\left(\times 10^{4}\right)\end{array}}{\mathrm{mol} \mathrm{l}^{-1}}$} & \multirow{2}{*}{$\frac{\text { Conversion }}{\%}$} & \multirow[t]{2}{*}{$\begin{array}{c}M_{\mathrm{cal}}{ }^{\mathrm{c}} \\
\left(\times 10^{-5}\right)\end{array}$} \\
\hline & & & & & & & \\
\hline \multirow[t]{4}{*}{ A } & DP-33 & Cumyl-K & 5 & 0.12 & 4.5 & & 0.30 \\
\hline & & & 4 & 0.11 & 3.6 & 92 & 0.62 \\
\hline & SP-33 & Cumyl-K & 5 & 0.17 & 6.1 & & 0.29 \\
\hline & & & 4.5 & 0.13 & 4.8 & 92 & 0.57 \\
\hline \multirow[t]{5}{*}{ B } & SDP-4 & Cumyl-K & 5 & 0.20 & 1.6 & & 1.3 \\
\hline & & & 3.5 & 0.039 & 1.3 & 97 & 1.6 \\
\hline & & & 8.5 & 0.16 & 1.1 & & 3.1 \\
\hline & SP-12 & Cumyl-K & 6 & 0.18 & 1.2 & & 1.6 \\
\hline & & & 10 & 0.14 & 1.0 & 100 & 3.1 \\
\hline \multirow[t]{5}{*}{ C } & SDP-1 & $n$-BuLi & 4 & 0.25 & 1.6 & & 1.6 \\
\hline & & & 2 & 0.038 & 1.3 & 70 & 1.9 \\
\hline & & & 3 & 0.15 & 0.9 & & 3.7 \\
\hline & SP-16 & Cumyl-K & 5.5 & 0.28 & 1.3 & & 2.2 \\
\hline & & & 12 & 0.10 & 1.1 & 100 & 3.2 \\
\hline \multirow[t]{11}{*}{ D } & DSP-1 & Cumyl-K & 4.5 & 0.064 & 2.0 & & 0.33 \\
\hline & & & 7.8 & 0.12 & 1.2 & 96 & 1.4 \\
\hline & & & 10.3 & 0.14 & 0.9 & & 3.0 \\
\hline & SP-15 & Cumyl-K & 4 & 0.19 & 1.3 & & 1.5 \\
\hline & & & 6 & 0.17 & 1.1 & 99 & 3.1 \\
\hline & SP-4 & $n$-BuLi & 5 & 0.18 & 1.8 & & 1.0 \\
\hline & & & 3 & 0.13 & 1.3 & 86 & 2.1 \\
\hline & SP-18 & Cumyl-K & 6 & 0.14 & 0.22 & & 6.6 \\
\hline & & & 9.3 & 0.12 & 0.17 & 95 & 14.0 \\
\hline & PS-1 & Cumyl-Cs & 6 & 034 & 1.3 & & 2.7 \\
\hline & & & 4 & 0.23 & 0.8 & 98 & 5.7 \\
\hline
\end{tabular}

a S, D and P denote styrene- $h_{8}$, styrene- $d_{8}$, and 2-vinylpyridine, respectively. The copolymerizations were carried out in the order of sample codes.

b The values in each section show the data for the precursor and final sample or for the 1st, 2nd precursors, and final sample consecutively.

c $M_{\text {cal }}$ are the molecular weights calculated from the amounts of monomers and initiator assuming $100 \%$ conversion. The initiator concentrations in the second and third copolymerizations were calculated by taking into account the amount of polymerization mixture sealed off in addition to the amount of monomer solution added. 


\section{RESULTS AND DISCUSSION}

Polymerization conditions and molecular characteristics of the block copolymers obtained are shown in Tables I and II. Each set in sections $A-D$ gives the data of a block copolymer labelled by styrene- $d_{8}$ and its reference block copolymer having approximately equal molecular weight and polystyrene content. The sample code SDP means that the copolymerization was carried out in the order of styrene- $h_{8}(\mathrm{~S})$, styrene- $d_{8}(\mathrm{D})$, and 2-vinylpyridine (P). All other sample codes have similar meanings. The two or three numbers in each experiment show the data for the precursor and final sample or for the 1st, 2nd precursors and final sample, consecutively from top to bottom. The experiments SP-4, SP-18, and PS1 were carried out to study the copolymerization mechanisms.

The initiator concentrations in Table I are the values in the polymerization mixtures, which were calculated from the concentration of the initiator determined by titration and the volume of the polymerization mixture. The values decrease with addition of the second or third monomer solution. The conversions were approximately estimated from the total amount of monomers used and the dry weight of the final sample. Table I shows that the conversions are close to $100 \%$ in all cases except SDP-1. The conversion of SDP-1 was low simply because the polymerization of 2-vinylpyridine was terminated before completion. $M_{\text {cal }}$ are the molecular weights of samples calculated from the amounts of monomers and initiator used, assuming $100 \%$ conversion in all cases. These values of $M_{\text {cal }}$ are found to agree with the observed molecular weights $\left(M_{n}\right.$ and $M_{w}$ ) in Table II.

Examples of GPC chromatograms of the block copolymers obtained and their precursors are shown in Figure 1. From these curves, it is clear that the molecular weight distributions of the samples as well as of their precursors show a single and narrow peak if they are
Table II. Molecular characteristics of block copolymers

\begin{tabular}{|c|c|c|c|c|c|}
\hline \multirow{2}{*}{$\begin{array}{c}\text { Sample } \\
\text { code }\end{array}$} & \multirow{2}{*}{$\begin{array}{c}M^{\mathrm{a}} \\
\left(\times 10^{-5}\right)\end{array}$} & \multirow{2}{*}{$\begin{array}{c}M_{w} / M_{n} \\
(\mathrm{GPC})\end{array}$} & \multicolumn{2}{|c|}{$C_{\mathrm{s}} / \mathrm{wt} / \mathrm{o}^{\mathrm{b}}$} & \multirow{2}{*}{$\frac{C_{\mathrm{d}}^{\mathrm{b}}}{\%}$} \\
\hline & & & M & $\mathrm{E}$ & \\
\hline DP-33 & 0.34 & 1.07 & & & \\
\hline A & 0.66 & 1.04 & 52 & - & 100 \\
\hline SP-33 & 0.37 & 1.10 & & & \\
\hline & 0.63 & 1.13 & 59 & - & 0 \\
\hline \multirow[t]{2}{*}{ SDP-4 } & 1.52 & 1.03 & & & \\
\hline & 1.97 & 1.06 & 49 & 50 & 19.1 \\
\hline B & $4.01^{*}$ & 1.08 & & & \\
\hline \multirow[t]{2}{*}{ SP-12 } & 2.11 & 1.04 & & & \\
\hline & 3.88 & 1.03 & 55 & 50 & 0 \\
\hline \multirow[t]{2}{*}{ SDP-1 } & 2.29 & 1.04 & & & \\
\hline & 2.78 & 1.05 & 74 & 71 & 19.4 \\
\hline $\mathrm{C}$ & 3.74 & 1.05 & & & \\
\hline \multirow[t]{2}{*}{ SP-16 } & 3.75 & 1.06 & & & \\
\hline & $5.23^{*}$ & 1.04 & 72 & 71 & 0 \\
\hline \multirow[t]{2}{*}{ DSP-1 } & 0.31 & 1.01 & & & \\
\hline & 1.37 & 1.05 & 58 & 48 & 22.7 \\
\hline D & 2.34 & 1.04 & & & \\
\hline \multirow[t]{2}{*}{ SP-15 } & 1.19 & 1.01 & & & \\
\hline & 2.43 & 1.06 & 49 & 48 & 0 \\
\hline \multirow[t]{2}{*}{ SP-4 } & 1.24 & 1.14 & & & \\
\hline & 2.45 & 1.11 & 51 & 50 & 0 \\
\hline \multirow[t]{2}{*}{ SP-18 } & $6.92^{*}$ & 1.07 & & & \\
\hline & $16.3^{*}$ & 1.17 & 43 & 48 & 0 \\
\hline
\end{tabular}

a The values without an asterisk are number-averaged molecular weights, while those with an asterisk are apparent weight-a veraged molecular weights.

${ }^{\mathrm{b}} \mathrm{Cs}$ and $\mathrm{Cd}$ are the total polystyrene $\left(h_{8}\right.$ and $\left.d_{8}\right)$ content in the sample and the styrene- $d_{8}$ content in the total polystyrene part, respectively. $\mathrm{M}$ and $\mathrm{E}$ show the total polystyrene content determined by molecular weight determination and elemental analysis, respectively.

polymerized in the order of styrene and 2vinylpyridine. The GPC chromatograms of the other samples polymerized in the order of styrene and 2-vinylpyridine also suggest narrow molecular weight distributions comparable to those in Figure 1, though they are not shown here. The values of $M_{w} / M_{n}$ in Table II were calculated from GPC chromatograms. 

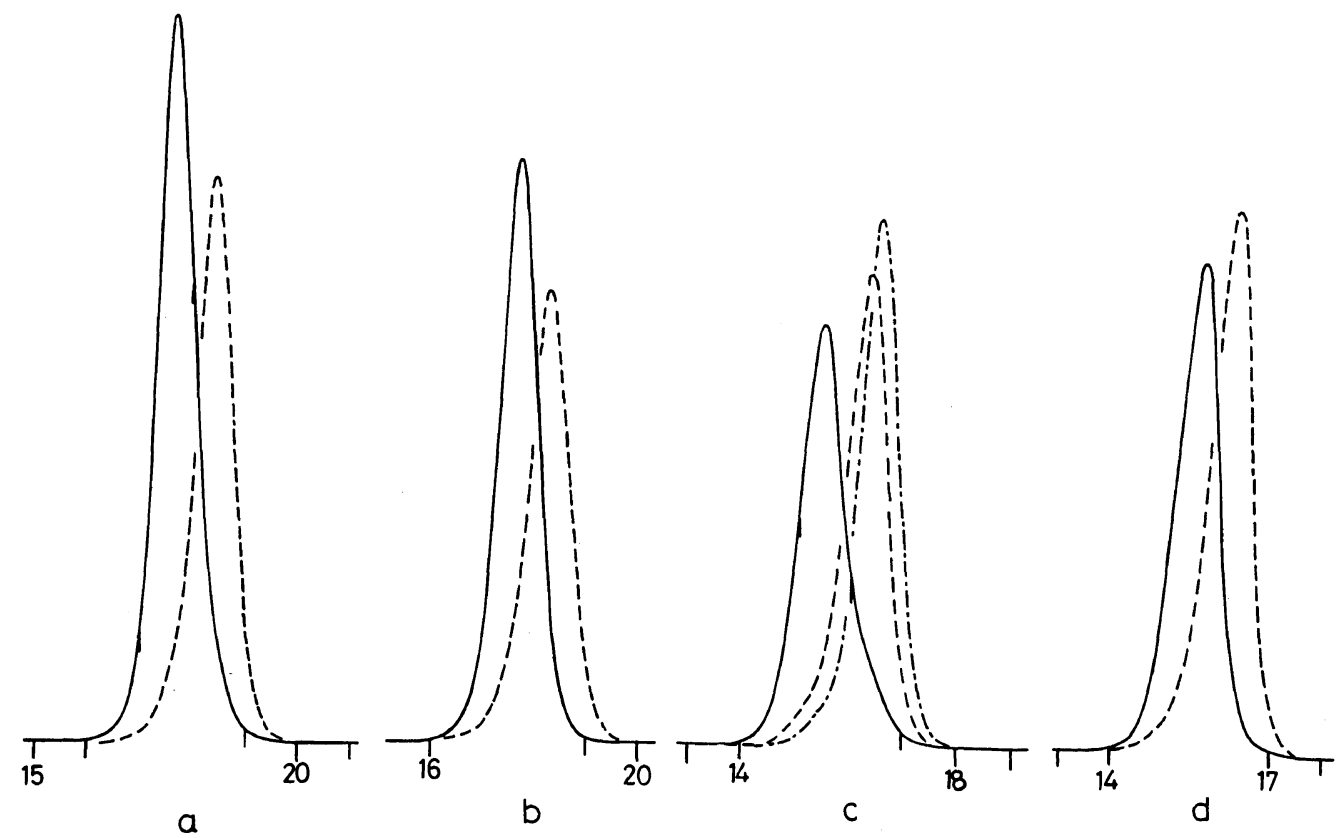

Figure 1. GPC chromatograms of di- and triblock copolymers (solid lines) and their precursors (dotted lines). a) DP-33; b) SP-33; c) SDP-4; d) SP-12.

They are close to unity. Since both molecular weight distributions of the final samples and their precursors are narrow, the chemical composition distributions of the block copolymers must also be narrow.

The styrene content of the block copolymers determined by molecular weight measurement (M) and also by elemental analysis (E) is shown in the fourth column of Table II. The agreement of both values is very good in most cases. The values of $\mathrm{Cd}$ show the styrene- $d_{8}$ content in the total styrene block.

From the data in Tables I and II and also in Figure 1, we may conclude that the block copolymerization of styrene- $h_{8}$ and $-d_{8}$ with 2vinylpyridine by the anionic polymerization method proceeds in a living manner. By comparing the data of the labelled samples with those of the reference samples in groups A$\mathrm{D}$, moreover, we may conclude that the preparation of block copolymers containing a poly(styrene- $d_{8}$ ) block can be carried out as satisfactorily as the block copolymerization

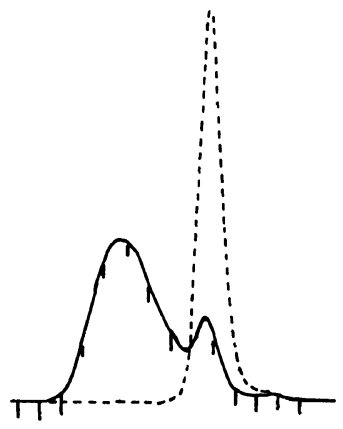

Figure 2. GPC chromatograms of PS-1 and its precursor (dotted line). Columns used are GMH6 $\times 2$.

between styrene- $h_{8}$ and 2-vinylpyridine in this range of molecular weight.

On the other hand, we can see in Figure 2 that the copolymerization in the order of 2vinylpyridine and styrene (PS-1) did not proceed satisfactorily. The GPC chromatogram of the precursor is a single and narrow peak but that of the block copolymer shows double peaks. Szwarc et al. pointed out that the rate of cross initiation of styrene monomer from poly- 
a

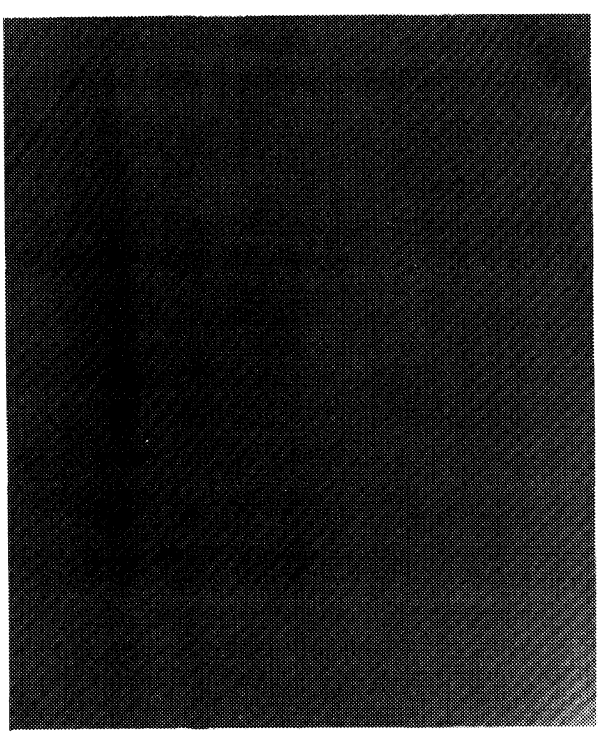

C

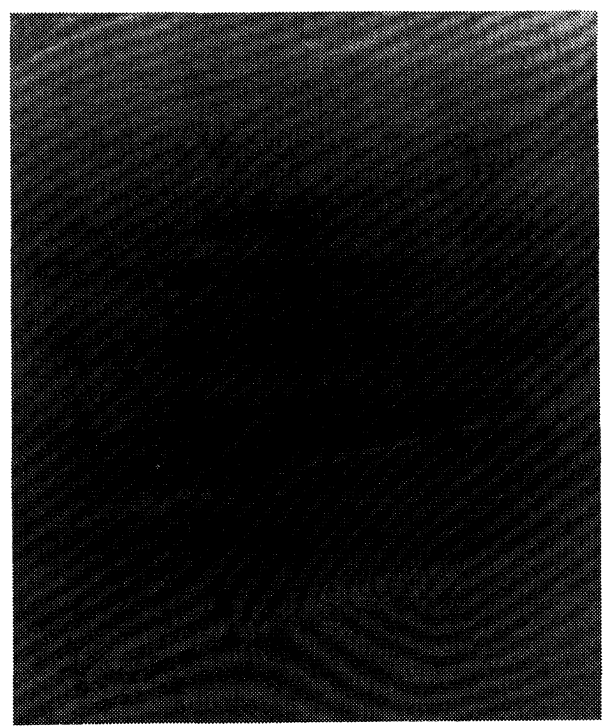

b $1000 \mathrm{~nm}$

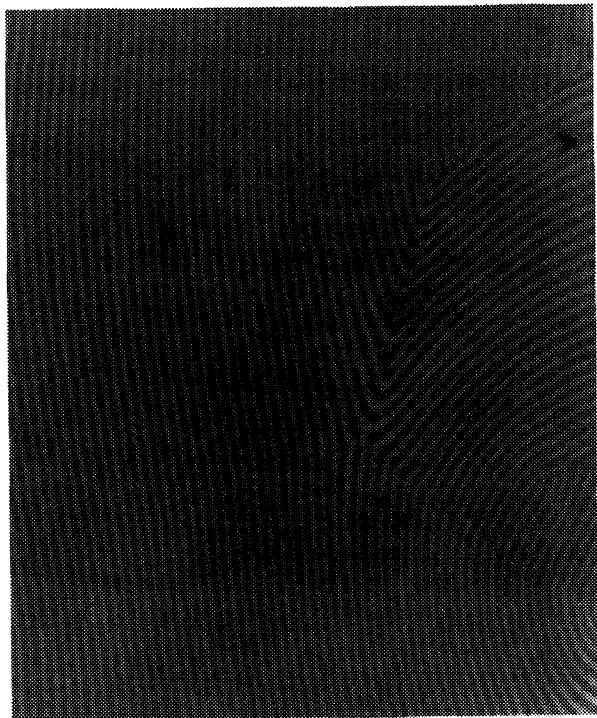

d $\quad 1000 \mathrm{~nm}$

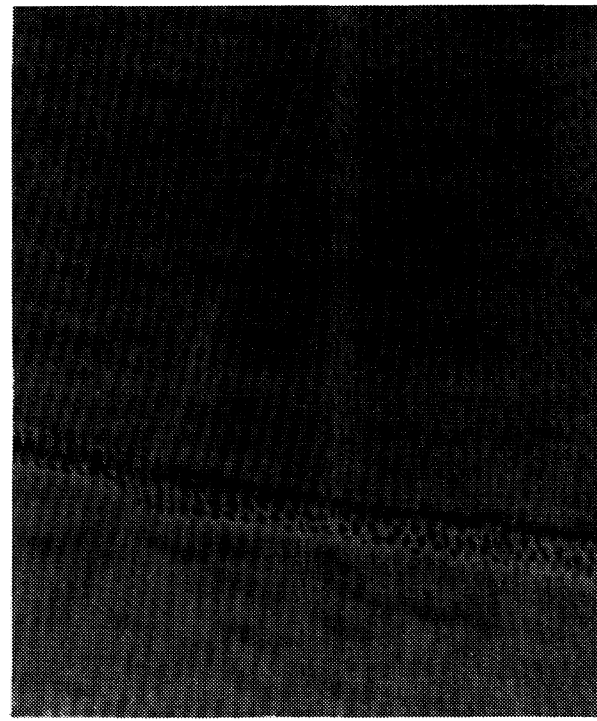

Figure 3. Electron micrographs of block copolymers. a) DP-33; b) SP-4; c) SDP-4; d) SDP-1.

(2-vinylpyridyl) anion is very slow compared ity that the living end of poly(2-vinylpyridyl) with that of 2-vinylpyridine from polystyryl anion. ${ }^{15}$ From the present experimental reanion is not unique.

Figure 3 shows electron micrographs of sults, moreover, we can point out the possibil- films of some samples cast from THF solu- 
tions. Their microphase separation structures are all lamellar except SDP-1. The lamellar structure is especially useful when we study the conformation of chains locating at interface by the small angle neutron scattering method.

Acknowledgements. We wish to thank Dr. Charles C. Han of the National Bureau of Standards, US Department of Commerce, for his helpful discussion and supplying us the styrene- $d_{8}$ monomer. We also acknowledge Dr. M. Fukuda of the Planning Research Institute, Toyo-Soda Manufacturing Co., Ltd. for taking the electron micrographs.

\section{REFERENCES}

1. Y. Matsushita, H. Furuhashi, H. Choshi, I. Noda, M. Nagasawa, T. Fujimoto, and C. C. Han, Polym. J., 14, 489 (1982).

2. Y. Matsushita, I. Noda, M. Nagasawa, T. Lodge, E. Amis, and C. C. Han, Macromolecules, 17, 1785 (1984).

3. K. Arai, T. Kotaka, Y. Kitano, and K. Yoshimura,
Macromolecules, 13, 1670 (1980).

4. Y. Matsushita, H. Choshi, T. Fujimoto, and M. Nagasawa, Macromolecules, 13, 1053 (1980).

5. H. Funabashi, Y. Miyamoto, Y. Isono, T. Fujimoto Y. Matsushita, and M. Nagasawa, Macromolecules 16, 1 (1983).

6. a) N. Kawabata and T. Tsuruta, Makromol. Chem., 86, 231 (1965); b) K. Hatada, T. Kitayama, S. Okahara, and H. Yuki, Polym. J., 13, 1045 (1981).

7. C. L. Lee, J. Smid, and M. Szwarc, Trans. Faraday Soc., 59, 1192 (1963).

8. P. D. Spiegelman and G. Parravano, J. Polym. Sci., $A, 2,2245$ (1964).

9. Y. Matsushita, K. Shimizu, Y. Nakao, H. Choshi, I. Noda, and M. Nagasawa, Polym. J., 18, 361 (1986).

10. T. Fujimoto (Department of Material Science and Technology, Technical University of Nagaoka, Nagaoka, Niigata, Japan), private communication.

11. T. Fujimoto, N. Ozaki, and M. Nagasawa, J. Polym. Sci., A, 3, 2259 (1965).

12. J. M. G. Cowie, R. Dey, and J. T. McCrindle, Polym. J., 2, 88 (1971).

13. S. Arichi, M. Yoshida, and Y. Ogawa, Polym. Prepr. Jpn., 23, 486 (1974).

14. H. Ohtani, S. Tsuge, Y. Matsushita, and $\mathbf{M}$. Nagasawa, Polym. J., 16, 727 (1984).

15. J. Smid and M. Szwarc, J. Polym. Sci., 61, 31 (1962). 